\title{
Editorial: Advances in spatial and temporal databases
}

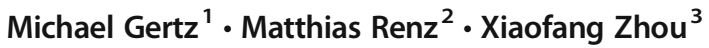

Published online: 19 October 2018

(C) Springer Science+Business Media, LLC, part of Springer Nature 2018

This special issue covers novel research contributions in the area of spatial and temporal data management, which has become again a hot area in the context of currently emerging fields such as urban analytics, smart transportation, smart mobility, and location-based systems and recommendation. It contains extended versions of the four contributions that have been selected as best papers at the 15th International Symposium on Spatial and Temporal Databases (SSTD 2017) in Arlington, VA, U.S.A., which took place in August 2017. The SSTD 2017 is the 15th event of a series of biannual events that discuss novel and exciting research in spatial, temporal and spatio-temporal data management and related technologies with the goal to set future research directions. SSTD symposia primarily focus on original results in theory, design, implementation, and applications of spatial and temporal database technology. They demonstrate how much spatial and temporal data are still popular and active research domains of interest, with many open research issues that need to be addressed and application areas to be explored. Over the past few years the range of topics addressed has evolved, with the emergence of big spatial and temporal data, location-based services, environmental and urban sensors, to mention but a few examples.

The selected papers published in this special issue are representatives of the recent developments of spatial-temporal data management. One important issue required in modern spatial data applications is to manage large amounts of spatial data. The paper by Louai Alarabi, Mohamed Mokbel and Mashaal Musleh (ST-Hadoop: A MapReduce Framework for Spatio-temporal Data) presents an extension of the Hadoop system to handle spatio-temporal

Matthias Renz

mr@informatik.uni-kiel.de; mrenz@gmu.edu

Michael Gertz

gertz@informatik.uni-heidelberg.de

Xiaofang Zhou

zxf@itee.uq.edu.au

1 Institute of Computer Science, Heidelberg University, Im Neuenheimer Feld 205, 69120 Heidelberg, Germany

2 Institute for Computer Science, Kiel University, Chrsitian-Albrechts-Platz 4, 24118 Kiel, Germany

3 Faculty of Engineering, Architecture and Information Technology, The University of Queensland, St Lucia, QLD, Brisbane 4072, Australia 
data and queries. The extensions cover multiple aspects such as the querying language, access methods, and query optimization.

For many spatial search applications, diversity in the set of answers is becoming a more and more important feature, specifically for recommendation systems. The paper by Camila Costa and Mario Nascimento (Towards Spatially- and Category-wise Diverse k-Nearest Neighbors Queries), which extends the SSTD-paper that has been recognized with the best paper award, studies the k-Diverse Nearest Neighbor problem that incorporates diversity into the traditional k-NN problem.

Another rather classic but still very important subfield in spatial and spatio-temporal database research is routing in traffic networks. The availability of multiple diverse context information associated with spatial entities provides many opportunities for advanced routing. In contrast to the traditional (rather simple) shortest path query, many more search criteria can be taken into consideration providing many interesting and relevant routing services. Liyue Fan, Luca Bonomi, Cyrus Shahabi, and Li Xiong address in their paper (Multi-User Itinerary Planning for Optimal Group Preference) the problem of path planning, in particular itinerary planning for multiple users. The paper considers planning the itinerary of a group of friends in a road network, taking into account their individual source and destination, the amount of deviation each of them can tolerate w.r.t. the shortest distance for their source-destination pair, and the individual type of facilities that each of them would want to visit along their joint itinerary.

The contribution provided by Zhipeng Huang and Nikos Mamoulis (Location-Aware Query Recommendation for Search Engines at Scale) addresses the problem of query recommendation, which has become a popular add-on feature of search engines. This work specially incorporates location information in the recommendation search process. Since a large percentage of search engine queries are issued from mobile devices one can provide better query recommendations by considering the physical locations of the query issuers.

We hope that these papers will inspire researchers to further conduct research in the many exciting areas of spatio-temporal data management.

Publisher's Note Springer Nature remains neutral with regard to jurisdictional claims in published maps and institutional affiliations. 